\section{Comparative analysis of targeted novel therapies in relapsed, refractory chronic lymphocytic leukemia}

Therapeutic options for chronic lymphocytic leukemia (CLL) patients have expanded over recent years as efficacious novel agents (NA) have received licensing approval, initially in relapsed, refractory $(\mathrm{R} / \mathrm{R})$ disease. Ibrutinib is a Bruton tyrosine kinase (BTK) inhibitor (BTKi) targeting Bcell receptor (BCR) pathway signaling. It has potent activity in relapsed disease as monotherapy ${ }^{1}$ with significant benefit compared to ofatumumab and combined with bendamustine-rituximab (BR) versus $\mathrm{BR}^{2}{ }^{2}$ Idelalisib also targets the BCR pathway by inhibiting the delta isoform of phosphoinositide 3-kinase (PI3Ki). It is licensed in combination with rituximab following improved progression-free survival (PFS) compared to rituximab in heavily pre-treated patients. ${ }^{3}$ However, toxicity concerns now limit its use in R/R CLL, and the randomized ASCEND trial $^{4}$ has shown PFS superiority for the second generation BTKi acalabrutinib versus idelalisib-rituximab (hazard ratio [HR] 0.29; $P<0.0001)$. Moreover, a large retrospective series has previously compared ibrutinib and idelalisib-rituximab as first NA (NA1) ${ }^{5}$ and found a similar, indirect PFS advantage for ibrutinib. Venetoclax is a potent, oral small-molecule BCL2 inhibitor (BCL2i) licensed as monotherapy \pm rituximab in R/R CLL. ${ }^{6,7}$ Venetoclax is increasingly utilized at first relapse in combination with rituximab for a 2-year fixed duration. ${ }^{6}$ However, to date, no prospective trials have directly compared ibrutinib with venetoclax as NA1 in R/R CLL. It remains a key unanswered question as to which of these two NA optimizes the balance of safety and efficacy when used as the NA1 in R/R CLL.

To address this, we report a large, international study to establish the efficacy of ibrutinib and venetoclax \pm anti-CD20 as NA1 in R/R CLL. To our knowledge, this is the largest series comparing these two approaches as NA1.

To address this question, a single NA database was created from prior international, collaborative, multicenter retrospective analyses examining each NA to describe and compare the characteristics and outcomes of CLL patients treated with ibrutinib or venetoclax \pm anti-CD20 as the NA1 between 2015-2019. To be eligible for comparison, patients could not have been treated with a targeted agent in the front-line setting and had to receive ibrutinib or venetoclax as NA1 no later than line 5 . Twenty centers participated in this study from academic and community sites

To define the study cohort for the primary analysis, medical chart reviews were performed to identify all con- secutive patients with CLL at each institution who received ibrutinib or venetoclax as NA1 in the R/R setting including only patients in routine clinical practice. To verify that cohorts were similar, baseline characteristics were collected prior to NA exposure. Using a standardized case report form, investigators collected data on preNA demographics, disease/prognostic characteristics (including del(17)p, complex karyotype [CK] defined: $\geq 3$ aberrations, IGHV status, and TP53 mutation), clinical and genetic characteristics, number and type of prior therapies. Toxicity data regarding dose interruptions, reductions, NA discontinuation, and reasons for discontinuation were collected.

Other primary endpoints were overall response rate (ORR) and PFS for the NA1. Investigators were requested to classify responses by International Workshop on CLL (iwCLL) criteria as complete remission (CR), partial remission (PR) (including PR with lymphocytosis), stable disease (SD), and progressive disease (PD). ${ }^{8}$ PFS was defined as the time from NA1 to last follow-up, progression of CLL or death. Overall survival (OS) was defined as the time from NA1 to death from any cause. PFS and OS were estimated by the Kaplan-Meier method. ${ }^{9}$ All other comparisons were descriptive. Comparisons were made using Cox regression or log-rank tests. Statistical analyses were performed using STATA 10.1. Follow-up was censored at most recent visit or death.

A total of 433 patients received a NA1: 385 ibrutinib; 48 venetoclax \pm anti-CD20 (80\% monotherapy). Data for PFS were available for 417 patients. Median follow-up was 14 months (ibrutinib) and 13.5 months (venetoclax), respectively. Each group had a median of two prior lines (range 1-4 for each). Median age of each cohort (ibrutinib: 69 years; venetoclax: 65 years) were similar, as were the proportion with adverse features such as del(17p) (Table 1).

Overall response rate and CR were: ibrutinib $(71 \%$ and $12 \%$, respectively) and venetoclax $(96 \%$ and $56 \%$, respectively). Venetoclax-treated patients had a statistically significantly higher $C R$ rate versus ibrutinib $(P<0.001)$. Figure 1A shows PFS of all patients stratified according to NA1. Using ibrutinib as the comparator (HR, 1.0), PFS HR for venetoclax was 0.29 (95\% Cl: 0.10-0.92; $P=0.036$ ) (Figure 1A). Using ibrutinib as the comparator for OS, HR was $1.2(95 \% \mathrm{Cl}: 0.47-3.1 ; P=0.70)$ for the venetoclax-treated cohort (Figure $1 \mathrm{~B})$. When considering del(17p), del(11q), CK and IGHV status, we could not identify a subgroup that benefited in terms of PFS when comparing each NA1 (data not shown). Considering the whole cohort, only those with a CK (HR, 1.99 [1.1-3.80]; $P=0.04$ ) had a statistically significant inferior PFS.

Dose interruptions were recorded in 33\% ibrutinib and $32 \%$ venetoclax-treated patients (Table 2 ). Dose reduc-

Table 1. Baseline characteristics of each novel therapy cohort.

\begin{tabular}{lcccc}
$\begin{array}{l}\text { Baseline characteristics } \\
\text { First novel therapy }\end{array}$ & $\mathrm{N}$ & Ibrutinib & $\mathrm{N}$ & $\begin{array}{c}\text { Venetoclax +/- anti-CD20 } \\
\text { Median age at treatment, }\end{array}$ \\
$\begin{array}{l}\text { years (range) } \\
\text { Median n. of prior therapies, }\end{array}$ & 382 & $69(27-95)$ & 48 & $2(1-4)$ \\
(range) & 385 & $2(1-4)$ & 48 & $34 \%$ \\
Del(17p) & 255 & $24 \%$ & 47 & $24 \%$ \\
Complex karyotype & 157 & $32 \%$ & 17 & $45 \%$ \\
Elevated LDH & 189 & $45 \%$ & 20 & 2 \\
\hline
\end{tabular}

N/n: number; $\mathrm{LDH}$ : lactate dehydrogenase. 
Letters to the Editor

trons were recorded in $22 \%$ of ibrutinib and $26 \%$ ventoclax-treated patients. Overall discontinuation rates were $41 \%$ ibrutinib and $25 \%$ venetoclax $(P=0.06)$. For ibrutinib, the most common discontinuation reasons were adverse events (AE) $(22 \%)$, CLL progression $(8 \%)$ and Richter's transformation (RT) $(2 \%)$. For venetoclax, the most common discontinuation reasons were allgeneic stem cell transplantation (10\%), CLL progression $(4 \%)$, and unrelated death event $(4 \%)$. RT was uncommon and comparable across cohorts (Table 2).

To our knowledge, this is the largest series comparing ibrutinib and venetoclax-based treatment in R/R CLL. In the first such analysis, we provide an indirect comparison of patients receiving ibrutinib and venetoclax as a NA1. We note that CR rates were higher with venetoclax treat- mont, which appeared to translate to a PFS advantage over ibrutinib but not an OS advantage. In light of this, and in the absence of randomized data comparing these approaches, our data provide reassurance that either option remains a reasonable approach as NA1 in R/R CLL. The PFS difference seen within this series may in part relate to the tolerability of each agent within routine clinical practice. Higher rates of discontinuation due to $\mathrm{AE}$ and frequent dose reductions/interruptions have been reported to be most pronounced in ibrutinib-treated patients ${ }^{10,11}$ and $\mathrm{AE}$ are the most dominant cause for ibrutinib discontinuation within our data.

Our study has several limitations. It is retrospective, and its conclusions remain hypothesis generating. However, in the absence of ongoing randomized trials

A

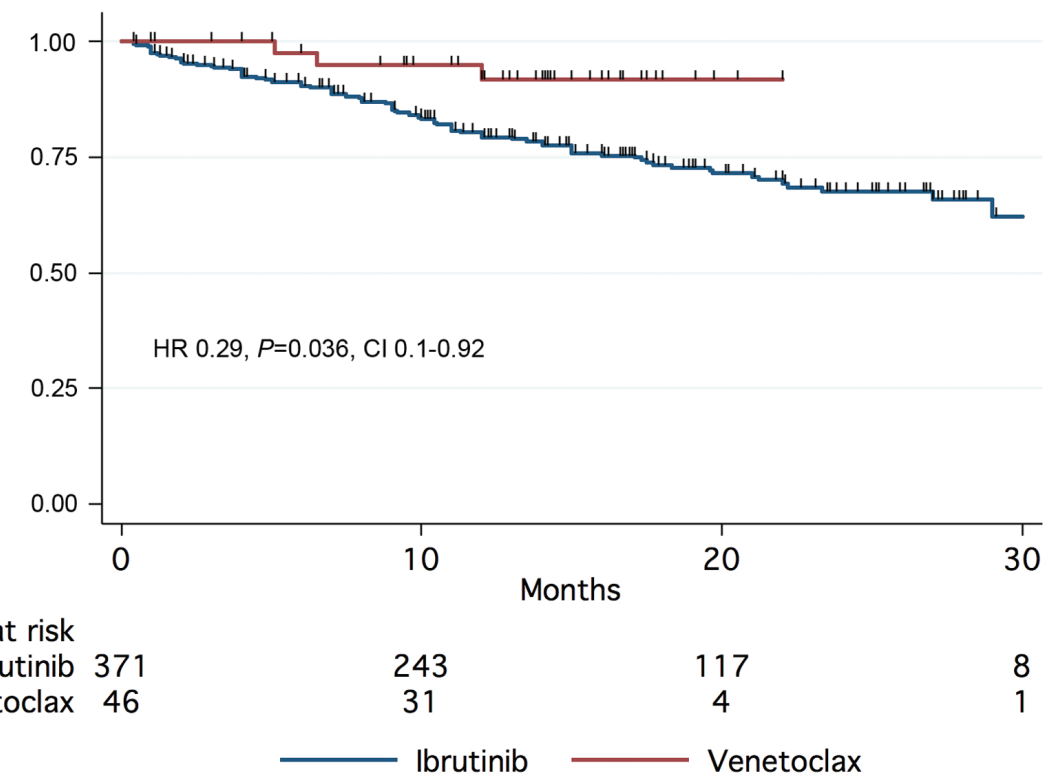

B

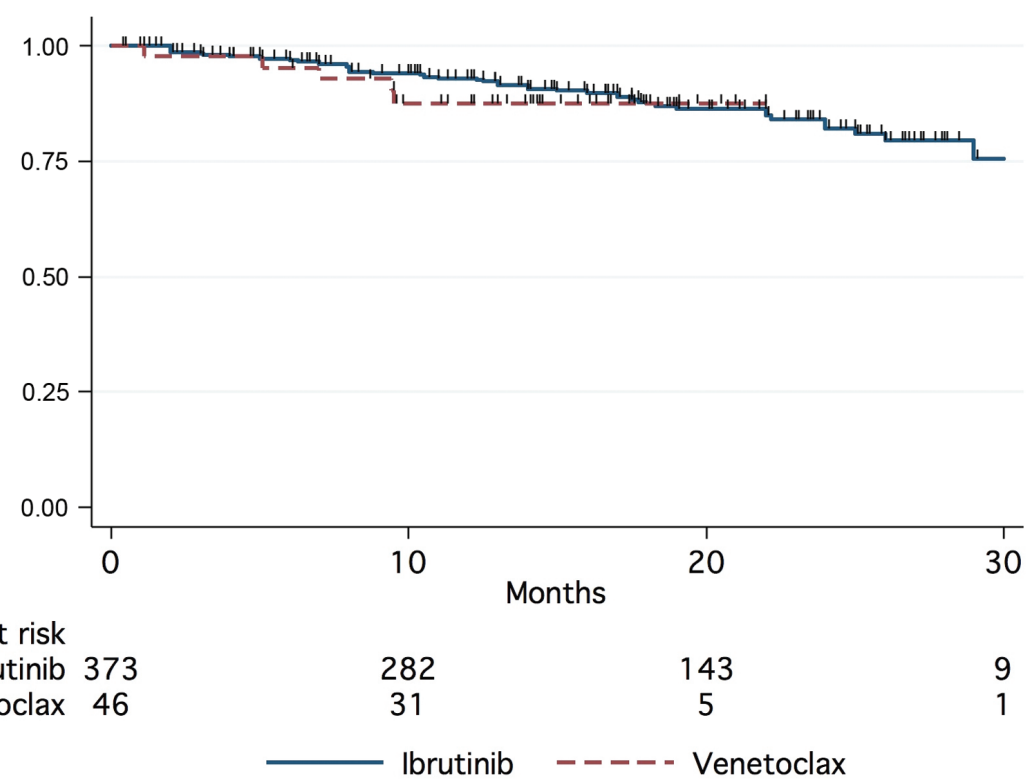

Figure 1. Survival outcome of patients with relapsed, refractory chronic lymphocytic leukemia CLL according to first novel agent received. (A) Progressionfree survival according to novel targeted agent. (B) Overall survival according to novel targeted agent. HR: hazard ratio; Cl: confidence interval.

haematological | 2021; 106(1)

285 
Table 2. Dosing patterns and discontinuations according to novel agent.

\begin{tabular}{lcc}
$\begin{array}{l}\text { Dosing patterns } \\
\text { First novel therapy }\end{array}$ & $\begin{array}{c}\text { Ibrutinito } \\
\text { Venetoclax +/- } \\
\text { anti-CD20 }\end{array}$ \\
\hline Dose reductions & $22 \%$ & $26 \%$ \\
\hline Dose interruptions & $33 \%$ & $32 \%$ \\
Discontinuation rate & $41 \%$ & $25 \%$ \\
\hline Most common discontinuation & Adverse & Allogeneic stem \\
reason & event & cell transplantation \\
Richter's transformation rate & $22 \%$ & $10 \%$ \\
\hline
\end{tabular}

comparing these two standard-of-care NA1 in CLL, our study may help to inform practice and aid future prospective trial design. We recognize the unavoidable biases associated with retrospective reporting, missing data, the lack of centralized response assessment and pathology review, and the potential for overestimating CR rates; however, any misclassification is likely to be non-differential. Information regarding dosing, interruptions, and toxicity were not prospectively collected leading to the risk of bias. To address this, we partnered with investigators with extensive experience in using NA and experienced in 'real-world' evidence collation. While the group has published similar datasets exploring NA sequencing, ${ }^{5,10-13}$ this analysis is the first published indirect comparison of ibrutinib and venetoclax in R/R CLL as first NA from our 'real-world' database and provides important new data in terms of NA sequencing and selection.

Patients within our series had received a median of two prior lines pre-NA, with a relatively high rate of del(17p) (25\% across all patients) and CK (31\% across all patients) for whom data were available. This likely reflects the fact that, early on, these agents were prescribed to heavily pretreated, poor-risk patients. Use of NA is quickly being brought forward to first relapse ${ }^{4}$ and front-line treatment. ${ }^{14,15}$ It is possible that survival analyses would produce different results in less heavily pre-treated patients. However, given the lack of prospective comparative data, we believe our comparison provides valuable information for healthcare providers in the decision-making process regarding which NA to use first in R/R CLL. Given the nature of this population, making inferences regarding front-line therapy is challenging and was not our primary goal. We also did not aim to specifically answer questions regarding subsequent sequencing beyond NA1; this work is currently ongoing. Finally, the patient numbers in the venetoclax group are small compared to the ibrutinib group and we acknowledge analyses may be underpowered to detect any possible differences. Although the baseline characteristics are similar, smaller cohorts could provide greater heterogeneity. The difference in baseline characteristics reflects prescribing practice over recent years where the strongest evidence for NA1 within trials ${ }^{1}$ and non-trial ${ }^{10,11}$ was initially formed with ibrutinib. Finally, our data were collected prior to recent analyses suggesting CK with $\geq 5$ aberrations selected the highest risk patients, and as such, our CK data should be interpreted in that light.

In conclusion, venetoclax and ibrutinib-based therapy as NA1 provide comparable OS outcomes in R/R CLL patients treated outside of trials. Our data suggest a significant PFS advantage for venetoclax-treated patients as
NA1, a finding that requires independent validation and reassessment for patients treated at first relapse outside of clinical trials. The selection of either as the NA1 should therefore be based on individual patient factors, drug access, deliverability, and patient preference.

Toby A. Eyre,,$^{{ }^{*}}$ Nicole Lamanna, ${ }^{2^{*}}$ Lindsey E. Roeker, Chaitra S Ujjani, ${ }^{4}$ Brian T. Hill, ${ }^{5}$ Paul M. Barr, ${ }^{6}$

Erick Lansigan, ${ }^{7}$ Bruce D. Cheson, ${ }^{8}$ Maryam Yazdy, John N. Allan, Joanna Rhodes, ${ }^{10}$ Stephen J. Schuster, Chadi Nabhan," Alan Skarbnik, ${ }^{12}$ Lorie Leslie, ${ }^{13}$

Prioty Islam, ${ }^{14}$ Katherine Whitaker, ${ }^{3}$ Catherine C. Coombs, ${ }^{15}$ Hande H. Tuncer, ${ }^{16}$ John M. Pagel, ${ }^{17}$ Ryan Jacobs, ${ }^{18}$ Allison M. Winter, ${ }^{5}$ Neil Bailey, ${ }^{17}$ Andrea Sitlinger, ${ }^{14}$ Anna H. Schuh, ${ }^{1}$ George Follows, ${ }^{19}$ Christopher P. Fox, ${ }^{20}$ Danielle M. Brander, ${ }^{14}$ Mazyar Shadman ${ }^{7}$ and Anthony R. Mato ${ }^{3}$

'Department of Haematology, Churchill Hospital, Oxford University Hospitals NHS Foundation Trust, Oxford, UK; ${ }^{2}$ Herbert Irving Comprehensive Cancer Center (New York-Presbyterian Columbia University Medical Center), New York, NY, USA; ${ }^{3}$ Memorial Sloan Kettering Cancer Center, New York, NY, USA; ${ }^{4}$ Seattle Cancer Care Alliance/Fred Hutchinson Cancer Research Center, Seattle, WA, USA; '5epartment of Hematology and Medical Oncology, Taussig Cancer Institute, Cleveland Clinic Foundation, Cleveland, OH, USA; ${ }^{6}$ Division of Hematology/Oncology, Wilmot Cancer Institute, University of Rochester, Rochester, NY, USA; ${ }^{7}$ Dartmouth-Hitchcock Medical Center, Lebanon, NH, USA; ${ }^{8}$ Georgetown University Hospital, Lombardi Comprehensive Cancer Center, Washington DC, USA; 'Weill Cornell Medicine, Long Island City, NY, USA; ${ }^{10}$ Division of Hematology and Oncology, Abramson Cancer Center, University of Pennsylvania, Philadelphia, PA, USA; ${ }^{11}$ Aptitude Health, Atlanta, GA, USA; ${ }^{12 N o v a n t ~ H e a l t h, ~ C h a r l o t t e, ~}$ NC, USA; ${ }^{13} J o h n$ Theurer Cancer Center at Hackensack University Medical Center, Hackensack, NJ, USA; ${ }^{14}$ Hematologic Malignancies and Cellular Therapy, Duke University Medical Center, Durham, NC, USA; ${ }^{15}$ Lineberger Comprehensive Cancer Center, University of North Carolina, Chapel Hill, NC, USA; ${ }^{16}$ Department of Medicine, Cancer Center, Tufts Medical Center, Boston, MA, USA; ${ }^{17}$ Center for Blood Disorders and Stem Cell Transplantation, Swedish Cancer Institute, Seattle, WA, USA; ${ }^{18}$ Department of Hematology, Lymphoma Division, Levine Cancer Institute, Charlotte, NC, USA; ${ }^{19}$ Department of Haematology, Addenbrookes, Cambridge, UK and ${ }^{20}$ Nottingham University Hospitals NHS Trust, Nottingham, UK

*TAE and NL contributed equally as co-first authors.

Correspondence:

TOBYA.EYRE - toby.eyre@ouh.nhs.uk

doi:10.3324/haematol.2019.241539

Disclosures: ARM holds a consultancy role for TG Therapeutics (in addition DSMB), Abbvie, Pharamacyclics, Johnson \& Johnson, Regeneron, Astra Zeneca, and Celgene and has received research funding from TG Therapeutics, Abbvie, Pharamacyclics, Johnson \& Johnson, Regeneron, Portola, DTRM, and Acerta; LER has received a travel grant from AbbVie; RJ has received honoraria from Genentech and AbbVie; BTH has served on advisory boards for and received research funding from Genentech/AbbVie; NL participated in advisory boards for AbbVie, AstraZeneca, Genentech, Gilead, Janssen, Juno, Pharmacyclics, is a member of a scientific advisory board for Celgene, and had research funding to the institution from AbbVie, Acerta, AstraZeneca, Beigene, Genentech, Gilead, Juno, Oncternal, TG Therapeutics, and Verastem; DMB serves as consultant for, is a member of the scientific advisory board of, and institution is the site of a PI clinical trial (grant paid to the institution) from AbbVie and Genentech; CSU has received consulting fees from AstraZeneca, Genentech, Gilead, and research support from AbbVie and Pharmacyclics; MSY received honoraria from Bayer as a speaker; APS has received research funding from BMS, has received consulting fees from Pharmacyclics, 
Janssen, AbbVie, Genentech, AstraZeneca, and serves on the Speakers Bureau for Pharmacyclics, Janssen, AbbVie, Genentech, Jazz Pharmaceuticals, Gilead, Kite Seattle, Genetics, Verastem, and Stock ownership for COTA healthcare; JMP has received consulting fees from AbbVie, Pharmacyclics, Genentech, Jazz Pharmaceuticals and serves on advisory boards for Pharmacyclics, AbbVie, and Genentech, speakers bureau for AbbVie, Pharmacyclics, Genentech, Jazz Pharmaceuticals, Gilead Sciences, Verastem, Kite, and Seattle Genetics; CCC has received honoraria from Loxo, Octapharma, $\mathrm{H3}$ Biomedicine, Pharmacyclics and AbbVie and has received consulting fees from AbbVie; $P M B$ has received consulting fees from AbbVie/PCYC, Gilead, Verastem, Genentech; CN is employed by Aptitude Health; BDC has received consulting fees from AbbVie, TG Therapeutics, Roche-Genentech, Pharmacyclics, Gilead, Epizyme, Celgene, Morhphosys, Astellas, AstraZeneca, Bayer, Karyopharm, receives research funding to the institution from AbbVie, TG Therapeutics, Roche-Genentech, Pharmacyclics, Gilead, Epizyme, Celgene, Trillium, AstraZeneca; TAE received Honora ria from Roche, Gilead, Abbvie, and Janssen, and holds an advisory board role for Gilead and has received research funding from Gilead, and travelled to conferences for Takeda, Abbvie, and Janssen; AG is an employee of COTA, Acerta, Genentech, Pharmacyclics/Janssen, and Kite/Gilead, reports receiving other commercial research support and speakers bureau honoraria from, and is a consultant/advisory board member for Takeda, Kite/Gilead, Pharmacyclics/Janssen, Genentech, and Acerta, and holds ownership interest (including patents) in COTA; CF reports receiving commercial research grants from Gilead, Abbvie, and Roche, reports receiving other commercial research support from Adienne, reports receiving speakers bureau honoraria from Abbvie, Celgene, Roche, and Janssen, and is a consultant/advisory boardmember for Abbvie, Celgene, Roche, Janssen, Sunesis, Gilead, Takeda, and Atarbio; no potential conflicts of interest were disclosed by the other authors.

Contributions: TE, NL, ARM, LR and MS designed the study; all authors performed data collection and analysis; TE wrote the manuscript and all authors reviewed it; ARM supervised the study.

Funding: this study was supported by funding from the Oxford NIHR Biomedical Research Centre. Views expressed are those of the authors and not necessarily those of the NHS or the NIHR or the United Kingdom's Department of Health.

\section{References}

1. Byrd JC, Furman RR, Coutre SE, et al. Targeting BTK with ibrutinib in relapsed chronic lymphocytic leukemia. N Engl J Med. 2013; 369(1):32-42

2. Chanan-Khan A, Cramer P, Demirkan F, et al. Ibrutinib combined with bendamustine and rituximab compared with placebo, bendamustine, and rituximab for previously treated chronic lymphocytic leukaemia or small lymphocytic lymphoma (HELIOS): a randomised, double-blind, phase 3 study. Lancet Oncol. 2016;17(2):200211.

3. Furman RR, Sharman JP, Coutre SE, et al. Idelalisib and rituximab in relapsed chronic lymphocytic leukemia. $N$ Engl J Med. 2014;370(11):997-1007.

4. Ghia P, Pluta A, Wach M, et al. Acalabrutinib vs rituximab plus idelalisib (IdR) or bendamustine (BR) by investigator choice in relapsed/refractory (RR) chronic lymphocytic leukemia: phase 3 ASCEND Study. Hematol Oncol. 2019;37(S2):86-87.

5. Mato AR, Hill BT, Lamanna N, et al. Optimal sequencing of ibrutinib, idelalisib, and venetoclax in chronic lymphocytic leukemia: results from a multi-center study of 683 patients. Ann Oncol. 2017; 28(5):1050-1056.

6. Seymour JF, Kipps TJ, Eichhorst B, et al. Venetoclax-rituximab in relapsed or refractory chronic lymphocytic leukemia. N Engl J Med. 2018;378(12):1107-1120.

7. Stilgenbauer S, Eichhorst B, Schetelig J, et al. Venetoclax in relapsed or refractory chronic lymphocytic leukaemia with $17 p$ deletion: a multicentre, open-label, phase 2 study. Lancet Oncol. 2016;17(6):768778.

8. Hallek M, Cheson BD, Catovsky D, et al. iwCLL guidelines for diagnosis, indications for treatment, response assessment, and supportive management of CLL. Blood. 2018;131(25):2745-2760.

9. Bland JM, Altman DG. Statistics notes: survival probabilities (the Kaplan-Meier method). BMJ. 1998;317(7172):1572.

10. UK CLL Forum. Ibrutinib for relapsed/refractory chronic lymphocytic leukemia: a UK and Ireland analysis of outcomes in 315 patients. Haematologica. 2016;101(12):1563-1572.

11. Mato AR, Nabhan C, Thompson MC, et al. Toxicities and outcomes of 616 ibrutinib-treated patients in the United States: a real-world analysis. Haematologica. 2018;103(5):874-879.

12. Mato AR, Thompson M, Allan JN, et al. Real-world outcomes and management strategies for venetoclax-treated chronic lymphocytic leukemia patients in the United States. Haematologica. 2018; 103(9):1511-1517.

13. Eyre TA, Kirkwood AA, Gohill S, et al. Efficacy of venetoclax monotherapy in patients with relapsed chronic lymphocytic leukaemia in the post-BCR inhibitor setting: a UK wide analysis. $\mathrm{Br}$ J Haematol. 2019;185(4):656-669.

14. Fischer K, Al-Sawaf O, Bahlo J, et al. Venetoclax and obinutuzumab in patients with CLL and coexisting conditions. N Engl J Med. 2019; 380(23):2225-2236.

15. Woyach JA, Ruppert AS, Heerema NA, et al. Ibrutinib regimens versus chemoimmunotherapy in older patients with untreated CLL. N Engl J Med. 2018;379(26):2517-2528. 